\title{
Adsorption of Poly(4-vinylpyridine) on Silica Surface
}

\author{
Masami KaWAguchi, Kenji KamiYA, and Akira TAKAHASHI \\ Department of Industrial Chemistry, Faculty of Engineering, \\ Mie University, Kamihamacho, Tsu, Mie 514, Japan.
}

(Received February 22, 1982)

\begin{abstract}
Adsorption of poly(4-vinylpyridine) onto a nonporous silica (Aerosil 130) from its nitroethane solution at $25^{\circ} \mathrm{C}$ was studied by IR spectroscopy and UV spectroscopy. The nitroethane was found to be essentially a theta solvent for poly(4-vinylpyridine). The observed frequency shift of the silanol groups for poly(4-vinylpyridine) is attributed to the hydrogen bonding between the nitrogen atoms of adsorbed poly(4-vinylpyridine) and silanol groups. Adsorbance $(A)$ was determined by UV spectroscopy, while the fraction of surface sites occupied $(\theta)$ and the fraction of adsorbed segments ( $p$ ) were determined by IR spectroscopy. The values of $A$ and $\theta$ in the plateau region increased with increasing molecular weight. The values of $p$ were independent of molecular weight. The data in the plateau region were compared with the theoreis of Roe, Silberberg, and Scheutjens and Fleer based on a lattice model, and qualitative agreement was found between theory and experiment.
\end{abstract}

KEY WORDS Adsorption / Poly(4-vinylpyridine)/ Silica / IR Spectroscopy / UV Spectroscopy / Frequency Shift / Adsorbance / Surface Sites / Adsorbed Segments / Adsorbed Amount /

Adsorption of a polymer onto a solid surface from its solution is governed by the polymersolvent, polymer-substrate, and solvent-substrate interactions. The latter two interactions depend not only on the chemical nature of both the polymer and solvent but also on the nature of the substrate. If the polymer-substrate interaction is stronger than the solvent-substrate interaction, the polymer is preferentially adsorbed on the substrate, and vice versa. Elucidation of these interactions is one of the central problems in the study of polymer adsorption. To this end, a well-characterized substrates should be used, such as silanol groups on the silica surface.

When functional groups of adsorbates interact with silanol groups on the silica surface, frequency shifts of infrared (IR) bands for silanol groups and functional groups of adsorbates are observed. ${ }^{1}$ Fontana and Thomas ${ }^{2}$ were the first to apply the IR method to the study of the adsorption of poly(lauryl methacrylate) onto a silica surface from its $n$ dodecane and cis-decalin solutions. They measured the surface sites occupied by polymer segments, $\theta$, and the fraction of polymer segments adsorbed, $p$, and discussed the interaction between the substrate and polymer segments. This IR spectroscopic technique has been extensively used to determine $\theta$ and $p$ for various polymers adsorbed onto a silica surface. ${ }^{3-16}$

In our previous papers, we studied the adsorption of polystyrene ${ }^{15}$ and polybutadiene ${ }^{16}$ onto the silica surface from their cyclohexane solutions at $35^{\circ} \mathrm{C}$ by IR spectroscopy, and compared the measured values of $\theta, p$, and the surface excess $\Gamma$ with several lattice model theories by choosing an appropriate value for the polymer-surface interaction parameter $\chi_{\mathrm{s}}$. Here, $\Gamma$ is defined by

$$
\Gamma=\left(A N_{\mathrm{A}} / n_{\mathrm{OH}} M_{\mathrm{u}}\right)
$$

where $A$ is the adsorbance, $N_{\mathrm{A}}$ is Avogadro's constant, $n_{\ominus \mathrm{OH}}$ is the number of silanol groups per unit area of silica surface, and $M_{\mathrm{u}}$ is the molecular weight of a monomer unit. For the adsorption of polystyrene,,$^{15}$ the frequency shift of the IR band of the silanol groups was attributed to the interaction between phenyl groups in the polymer and the silanol groups, while for the adsorption of polybutadiene, ${ }^{16}$ it was attributed to the interaction 
between double bonds in the polymer and the silanol groups.

Since frequency shifts of IR bands for adsorbed pyridine derivatives, ${ }^{1,17}$ and for the silanol groups ${ }^{17}$ have been observed, the nitrogen atoms of adsorbed pyridine derivatives must be hydrogen-bonded to the silanol groups of the silica surface. Thies, Peyser, and Ullman ${ }^{3}$ investigated the adsorption of poly(4vinylpyridine) onto the silica surface from its chloroform solution using IR spectroscopy and determined $p$ and $\Gamma$. However, they did not compare the experimenal data of $p$ and $\Gamma$ with relevant theories. In this paper, we report an IR study on the adsorption of poly(4-vinylpyridine) onto the Aerosil silica 130 from its nitroethane solution at $25^{\circ} \mathrm{C}$, which is close to a theta solvent for this polymer. The data of $\Gamma, \theta$, and $p$ are compared with the theories of Roe ${ }^{18}$ and Silberberg, ${ }^{19}$ and Scheutjens and Fleer. ${ }^{20}$

\section{EXPERIMENTAL}

\section{Materials}

Poly(4-vinylpyridine) (PVPy) was prepared by solution polymerization of freshly distilled 4-vinylpyridine in benzene at $70^{\circ} \mathrm{C}$ for $8 \mathrm{~h}$ using AIBN as an initiator. PVPy was precipitated by ethyl acetate, dried in a vacuum, dissovled in nitroethane, and separated into nine fractions using benzene at $25^{\circ} \mathrm{C}$ as a precipitant. The intrinsic viscosities in ethanol at $25^{\circ} \mathrm{C}^{21}$ were measured by a Ubbelhode viscometer and the molecular weights of the fractions were calculated from

$$
[\eta]=2.5 \times 10^{-4} M^{0.68}
$$

Three fractions were selected for the present measurements. Their intrinsic viscosities in nitroethane at $25^{\circ} \mathrm{C}$ were also measured and are given in Table I.

Organic solvents were purified by distillation. In particular, nitroethane for adsorption experiments

Table I. Characteristics of poly(4-vinylpyridine)

\begin{tabular}{lrc}
\hline \multicolumn{1}{c}{ Samples } & \multicolumn{1}{c}{$M$} & {$[\eta] / \mathrm{dl} \mathrm{g}^{-1}$} \\
\hline PVPy-1 & $6.7 \times 10^{4}$ & 0.290 \\
PVPy-2 & $14.0 \times 10^{4}$ & 0.410 \\
PVPy-3 & $31.1 \times 10^{4}$ & 0.682 \\
\hline
\end{tabular}

was distilled three times just before use.

The nonporous Aerosil 130 silica supplied from Degussa A. G. (West Germany) was used as the adsorbent after being cleaned it by the procedure described in our previous papers. ${ }^{15,16}$ The concentration of surface silanol groups was determined by the method of Boehm and Schneider ${ }^{22}$ to be three silanol groups per $100 \AA^{2}$. According to the manufacturer, the particle diameter was $160 \AA$ with a surface area of $141 \mathrm{~m}^{2} \mathrm{~g}^{-1}$.

\section{Adsorption of PVPy onto the Silica}

$25 \mathrm{ml}$ of PVPy nitroethane solution of known concentration were mixed with the silica (usually about $0.20 \mathrm{~g}$ ) in a stoppered centrifuge tube and the mixture was gentlely stirred by a magnetic chip for $20 \mathrm{~h}$ at $25^{\circ} \mathrm{C}$. The silica suspensions were centrifuged at 5,000 $\mathrm{g}$ for $20 \mathrm{~min}$ to sediment the silica and then the supernatant was carefully removed. Following the evaporation of nitroethane, the residue was dried under vaccum, dissolved in methanol, and its concentration was measured at a wavelength of $260 \mathrm{~nm}$ with a UV spectrometer. The relevent concentration of PVPy was determined from a calibration curve. Adsorbance, $A\left(\mathrm{~g} \mathrm{~cm}^{-2}\right.$ of silica), was determined by the difference between the amount of the PVPy initially added and that in the supernatant, and also from the amount of the silica added.

IR measurements on the sedimented silica and the silica immersed in 4-vinylpyridine monomer were carried out according to a method similar to that described previously. ${ }^{15,16}$

The method for calculating $\theta$ and $p$ is described in our previous papers. ${ }^{15,16}$

\section{RESULTS}

\section{Adsorbance}

The adsorption isotherms of the samples studied are shown in Figure 1. Initially, they rose steeply with increasing bulk equilibrium polymer concentration $C_{\mathrm{p}}$ and reached a plateau region. The measured values of adsorbance, $A$ are summarized in Table II for all samples. The value of $A$ in the plateau region increased with increasing molecular weight.

Fraction of Surface Sites Occupied, $\theta$

The differential infrared spectrum between the 
Table II. Adsorption data for poly(4-vinylpyridine) on Aerosil 130

\begin{tabular}{|c|c|c|c|c|c|c|}
\hline \multirow[t]{2}{*}{ Samples } & \multirow{2}{*}{$\begin{array}{c}\text { Bulk } \\
\text { equilibrium } \\
\text { concentration, } \\
\frac{C_{\mathrm{p}} \times 10^{2}}{\mathrm{~g} \mathrm{ml^{-1 }}}\end{array}$} & \multirow{2}{*}{$\begin{array}{l}\text { Adsorbance, } \\
\frac{A \times 10^{7}}{\mathrm{~g} \mathrm{~cm}^{-2}}\end{array}$} & \multirow{2}{*}{$\begin{array}{c}\text { Fraction of } \\
\text { segments } \\
\text { adsorbed, } \\
p\end{array}$} & \multirow{2}{*}{$\begin{array}{c}\text { Fraction of } \\
\text { surface sites } \\
\text { occupied, } \\
\theta\end{array}$} & \multicolumn{2}{|c|}{ Surface excess } \\
\hline & & & & & $\Gamma$ & $\Gamma=\theta / p$ \\
\hline \multirow[t]{5}{*}{ PVPy-1 } & 0.001 & 0.48 & 0.38 & 0.39 & 0.92 & 1.03 \\
\hline & 0.0044 & 0.73 & 0.30 & 0.44 & 1.40 & 1.47 \\
\hline & 0.0109 & 0.90 & 0.26 & 0.45 & 1.73 & 1.73 \\
\hline & 0.0830 & 1.34 & 0.20 & 0.49 & 2.55 & 2.45 \\
\hline & 0.294 & 1.58 & 0.15 & 0.45 & 3.02 & 3.00 \\
\hline \multirow[t]{5}{*}{ PVPy-2 } & 0.0030 & 0.26 & 0.66 & 0.35 & 0.43 & 0.53 \\
\hline & 0.0107 & 0.48 & 0.50 & 0.45 & 0.91 & 0.90 \\
\hline & 0.0265 & 1.06 & 0.20 & 0.43 & 2.01 & 2.15 \\
\hline & 0.209 & 1.73 & 0.15 & 0.51 & 3.31 & 3.40 \\
\hline & 0.282 & 1.76 & 0.15 & 0.50 & 3.37 & 3.33 \\
\hline \multirow[t]{5}{*}{ PVPy-3 } & 0.0016 & 0.33 & 0.60 & 0.41 & 0.62 & 0.68 \\
\hline & 0.0075 & 0.42 & 0.41 & 0.50 & 0.81 & 0.97 \\
\hline & 0.0380 & 0.96 & 0.40 & 0.51 & 1.83 & 1.28 \\
\hline & 0.147 & 1.71 & 0.15 & 0.46 & 3.26 & 3.07 \\
\hline & 0.240 & 1.95 & 0.15 & 0.54 & 3.73 & 3.60 \\
\hline
\end{tabular}

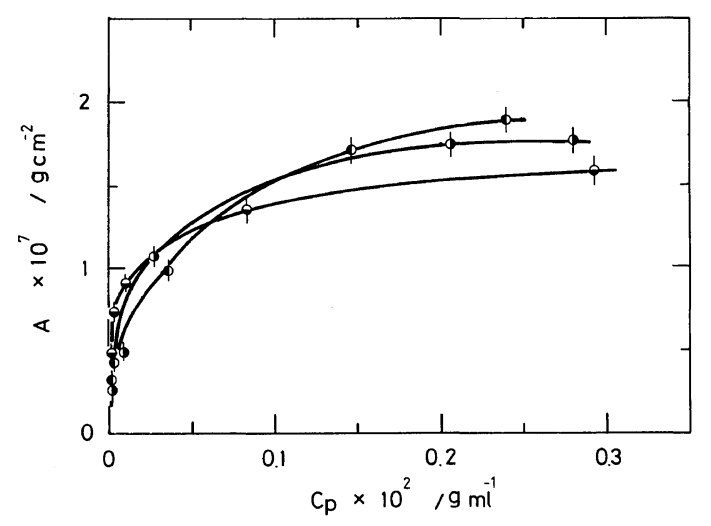

Figure 1. Adsorption isotherms of poly(4-vinylpyridine)s: $\odot$, PVPy-1; 1 , PVPy-2; 1 , PVPy-3.

supernatant PVPy solution and the silica on which PVPy was adsorbed shows two characteristic bonds in Figure 2: one located at $3633 \mathrm{~cm}^{-1}$, due to the isolated silanol groups and the other at $3465 \mathrm{~cm}^{-1}$, due to the hydrogen bonding between the silanol groups and the nitrogen atoms of the adsorbed PVPy. Thus, both the number of free silanol groups and that of the occupied silanol groups can be determined provided the extinction coefficients for the respective groups are known.

For the free silanol groups on the silica immersed

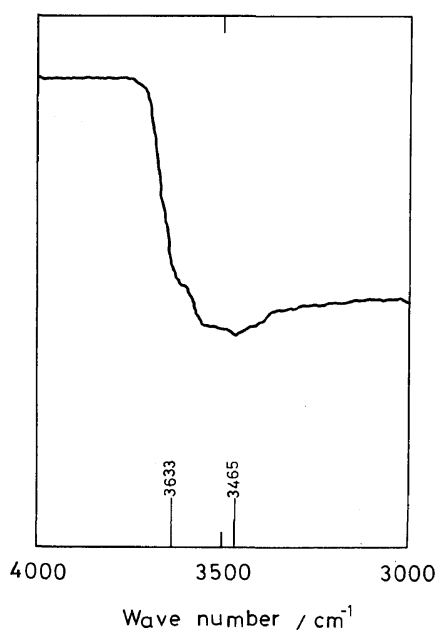

Figure 2. IR-spectrum of Aerosil 130 adsorbed poly(4vinylpyridine).

in nitroethane (Figure 3 ) the extinction coefficient at $3633 \mathrm{~cm}^{-1}$ was determined to be $54.91 \mathrm{~mol}^{-1} \mathrm{~cm}^{-1}$.

The IR spectrum of the silica immersed in 4vinylpyridine monomers is also shown in Figure 3. The appearance of a broader band at $3375 \mathrm{~cm}^{-1}$ may be attributed to the interaction between the nitrogen atoms of the monomer and the silanol groups on the silica surface. The shift due to the 


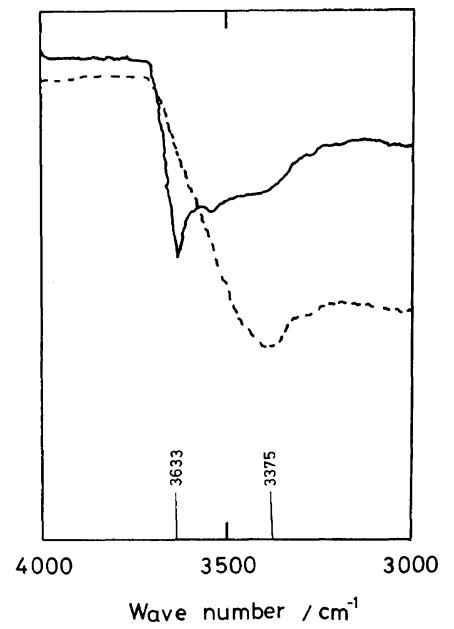

Figure 3. IR-spectra of Aerosil 130 immersed in (nitroethane and (----) 4-vinylpyridine monomer.

monomer is greater than that due to PVPy, probably because the strength of the interactions of the silanol groups with the nitrogen atoms in the monomers and those in PVPy are different. However, we have assumed that the extinction coefficient of $144.51 \mathrm{~mol}^{-1} \mathrm{~cm}^{-1}$ at $3465 \mathrm{~cm}^{-1}$ for the nitrogen atoms in PVPy is equal to that in the monomer at $3375 \mathrm{~cm}^{-1}$.

The value of $\theta$ can be calculated from

$$
\theta=S_{3,465} /\left(S_{3,465}+S_{3,633}\right)
$$

where $S_{3,465}$ and $S_{3,633}$ are the number of silanol groups adsorbed by PVPy and that of free silanol groups, respectively. The values of $\theta$ calculated from equation 3 are listed in Table II. These values increase with the increasing bulk equilibrium concentration $C_{\mathrm{p}}$ for each sample. The limiting value of $\theta$ in the plateau region increases with an increase in molecular weight.

\section{Fraction of Segments Adsorbed, $p$}

The values of $p$ obtained are summarized in Table II. These values decrease with increasing bulk equilibrium concentration for each sample. The $p$ values in the plateau region are constant, being 0.15 for all samples.

\section{DISCUSSION}

\section{Surface Excess, $\Gamma$}

The surface excess $\Gamma$, the total number of seg- ments adsorbed per lattice site, is an important quantity, since it can be regarded as the adsorbed amount per lattice site. The values of $\Gamma$ calculated from measured $A$ are compared with the values of $\theta / p$ in Table II. We find that the values of $\Gamma$ determined by the two methods are consistent.

\section{Comparison of Experimental Results and Theory}

Several lattice model theories for polymer adsorption are available for comparison with the experimental quantities of $\theta, p$, and $\Gamma$ : Roe's theory ${ }^{18}$ specifies no particular conformations for adsorbed polymers; Silberberg's theory ${ }^{19}$ assumes the loop-train conformation; and Scheutjens and Fleer's theory ${ }^{20}$ is based on the loop-traintail conformation. In these theories, the values of $\theta, p$, and $\Gamma$ are calculated as a function of the volume fraction, $\phi^{*}$, the degree of polymerization, $n$, the Flory-Huggins paramerer, $\chi$, and the polymer-surface interaction parameter, $\chi_{\mathrm{s}}$, while, in Silberberg's theory, a flexibility parameter $\gamma_{\mathrm{s}} \gamma_{\mathrm{B}}$ is necessary as an additional variable.

In making calculations with Roe's theory, we used the computer program provided through the courtesy of Professor Roe, and when using the theories of Silberberg and Scheutjens and Fleer, reference was made to the published results of the authors, a procedure found to be more exact and difficult.

The $\chi$ parameter for PVPy-nitroethane at $25^{\circ} \mathrm{C}$ was determined to be $0.49_{8}$ from thermodynamic analysis using the Stockmayer-Fixman plot for the intrinsic viscosity data in Table I. Thus, we assume nitroethane to be a theta solvent. Therefore, except for $\chi_{\mathrm{s}}$ and $\gamma_{\mathrm{s}} \chi_{\mathrm{B}}$, all the quantities were known. We attempted to compare the polymerization degree dependence of $\theta, p$, and $\Gamma$ with that calculated by the theories of Roe, Silberberg, and Scheutjens and Fleer.

In Figures 4, 5, and 6, the polymerization degree dependence of $\theta, p$, and $\Gamma$ in the plateau region is compared with that calculated by the theories of Roe, Silberberg, and Scheutjens and Fleer. For comparison, we selected $\chi=0.5$ and $\phi^{*}=10^{-3}$ in the theories of Roe and of Scheutjens and Fleer, and $\chi=0.5, \phi^{*}=10^{-3}$, and $\gamma_{\mathrm{s}} \gamma_{\mathrm{B}}=0.1$ in the theory of Silberberg. However, the results from the theory of Scheutjens and Fleer are available only at a degree of polymerization of $10^{3}$ as a function of $\chi_{\mathrm{s}}$, while results using the theory of Silberberg are 


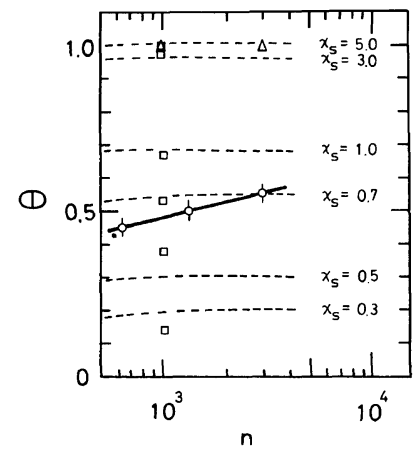

Figure 4. Degree of polymerization dependence of $\theta$. The dashed lines were calculated by the theory of Roe ${ }^{18}$ for different values of polymer-surface interaction parameter, $\chi_{\mathrm{s}}$. The triangles were calculated by the theory of Silberberg ${ }^{19}$ for $\chi_{\mathrm{s}}=\infty$. The squares were calculated by the theory of Scheutjens and Fleer ${ }^{20}$ for different $\chi_{\mathrm{s}}$ values: $\chi_{\mathrm{s}}$ values are $5.0,3.0,1.0,0.7$, and 0.5 from top to bottom.

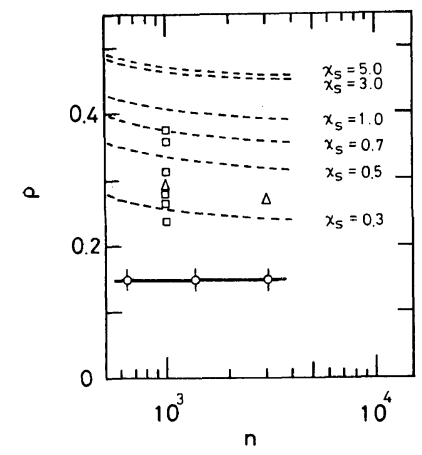

Figure 5. Degree of polymerization dependence of $p$. The dashed lines were calculated by the theory of Roe ${ }^{18}$ for different values of polymer-surface interaction parameter; $\chi_{\mathrm{s}}$. The triangles were calculated by the theory of Silberberg ${ }^{19}$ for $\chi_{\mathrm{s}}=\infty$. The squares were calculated by the theory of Scheutjens and Fleer ${ }^{20}$ for different $\chi_{\mathrm{s}}$ values: $\chi_{\mathrm{s}}$ values are $5.0,3.0,1.0,0.7$, and 0.5 from top to bottom.

available at polymerization degrees of $10^{3}$ and $3 \times 10^{3}$ for $\chi_{\mathrm{s}}=\infty$, namely $\theta=1$. The values calculated by the theory of Scheutjens and Fleer are shown by squares and those by the theory of Silberberg are shown by triangles.

By the theories of Roe, Scheutjens and Fleer, the calculated values of $\theta, p$, and $\Gamma$ increase with increasing $\chi_{\mathrm{s}}$, but, above $\chi_{\mathrm{s}}=5.0, \theta, p$, and $\Gamma$ remain constant. The measured values of $\theta$ increase as the degree of polymerization increases. The $\theta$ calculated

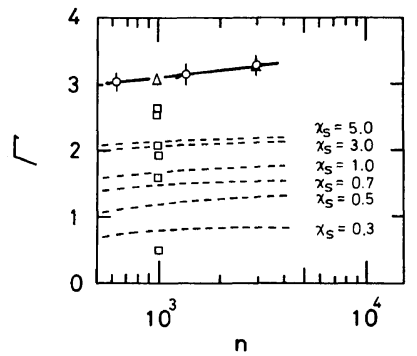

Figure 6. Degree of polymerization dependence of $\Gamma$. The dashed lines were calculated by the theory of Roe ${ }^{18}$ for different values of polymer-surface interaction parameter, $\chi_{s}$. The triangles were calculated by the theory of Silberberg ${ }^{19}$ for $\chi_{s}=\infty$. The squares were calculated by the theory of Scheutjens and Fleer $^{20}$ for different $\chi_{\mathrm{s}}$ values: $\chi_{\mathrm{s}}$ values are 5.0, 3.0, 1.0, 0.7, and 0.5 from top to bottom.

by the theory of Roe slightly increases with the degree of polymerization and the value for $\chi_{\mathrm{s}}=0.7$ is in good agreement with the measured $\theta$. Above $\chi_{\mathrm{s}}=0.7$ the $\theta$ calculated by this theory is nearly equal to that by the theory of Scheutjens and Fleer.

Measured $p$ are almost independent of the degree of polymerization. The $p$ calculated by the theory of Roe decrease slowly with increasing polymerization degree for all values of $\chi_{\mathrm{s}}$ and are much larger than the measured $p$. The $p$ calculated by the theory of Roe are larger than those by the theory of Scheutjens and Fleer for the same $\chi_{\mathrm{s}}$. The $p$ calculted by the theory of Silberberg are about twice as large as the measured $p$.

Measured $\Gamma$ increase with increasing degree of polymerization. The $\Gamma$ calculated by the theory of Roe decrease slowly with increasing polymerization but even for $\chi_{\mathrm{s}}=5.0$ the calculated value is smaller than the measured $\Gamma$. The $\Gamma$ calculated by the theory of Scheutjens and Fleer for $\chi_{\mathrm{s}}=5.0$ approach the measured values but there was no complete agreement between calculated and measured $\Gamma$. The $\Gamma$ calculated by the theory of Silberberg are in good agreement with the measured values. However, the condition $\chi_{\mathrm{s}}=\infty$ is too extreme.

Based on the above comparisons it becomes evident that there is yet no theory which provides an adequate explanation for the three experimental values of $\theta, p$, and $\Gamma$ when a particular value of $\chi_{\mathrm{s}}$ parameter is assumed. However, the fact is important that the theories based on lattice model explain qualitatively the degree of polymerization depen- 
dence of $\theta, p$, and $\Gamma$. The discrepancy between theory and experiment is nearly the same for each of the three theories examined. Thus, we cannot make any conclusion in regard to the predominant conformation using only the data of $\theta, p$, and $\Gamma$.

Degree of Polymerization Dependence of $\Gamma, p$, and $\theta$

The molecular weight $(M)$ dependence of adsorbance $A$ in the plateau region is ordinarily represented by the empirical equation, ${ }^{23}$

$$
A=K M^{\alpha}
$$

where $K$ and $\alpha$ are constants. The constant $\alpha$ bears some relation to the conformation of the adsorbed polymer chains. However this relation should be treated carefully when the molecular weight dependence of the thickness of the adsorbed layer is unknown. $\mathrm{We}^{24}$ have pointed out that it is misleading to make an inferrence about polymer conformations only from the value of $\alpha$, on the basis of a comparison of the adsorption of polystyrenes onto the chrome plates at the theta point with theory.

In this study, unique values of $\Gamma$ were obtained since a well-characterized silica surface was used as the adsorbent. In Figure 7, all the available data for $\Gamma$ in the plateau region under the theta condition, being data from the present experiment and those by Kawaguchi, Hayakawa, and Takahashi ${ }^{15}$ and by Linden and Leemput ${ }^{14}$ for polystyrene in cyclohexane at $35^{\circ} \mathrm{C}$ are plotted against the degree of polymerization instead of the molecular weight. For comparison, the $\Gamma$ values of polybutadiene adsorbed on the silica surface in cyclohexane, ${ }^{16}$ and those of polystyrene adsorbed on the silcia surface in carbon tetrachloride, ${ }^{14}$ using good solvents for polybutadiene and polystyrene, are also included in Figure 7. It is apparent that the $\Gamma$ values for the theta solvents fall on a master curve independent of the polymer sample and solvent. While the $\Gamma$ values for good solvents are located below the master curve. Though the available data under the theta condition are limited at the present to these three cases, the

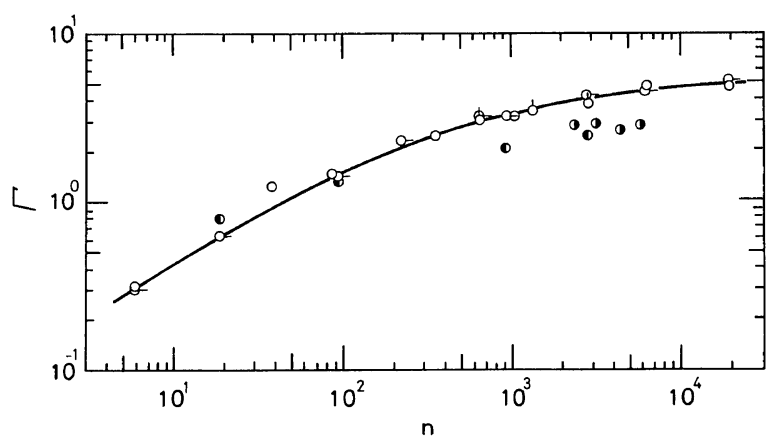

Figure 7. Plot of $\Gamma$ vs. the degree of polymerization: $\delta$, poly(4-vinylpyridine)s measured in this experiment; $O$, polystyrenes in cyclohexane measured by Kawaguchi, Hayakawa, and Takahashi $\left({ }^{15}\right)$; $\mathrm{O}$-, polystyrenes in cyclohexane measured by Linden and Leemput ${ }^{14} ; \boldsymbol{O}$, polybutadienes in cyclohexane measured by Kawaguchi, Sano, and Takahashi ${ }^{16} ; \mathbf{D}$, polystyrene in carbon tetrachloride measured by Linden and Leemput. ${ }^{14}$

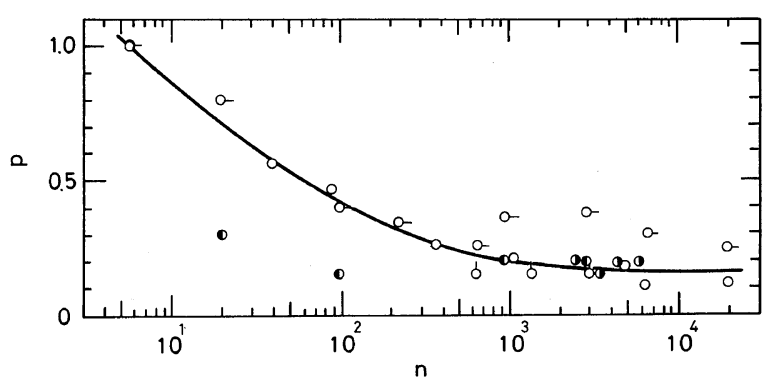

Figure 8. Plot of $p$ vs. the degree of polymerization. Symbols are the same as in Figure 7. 


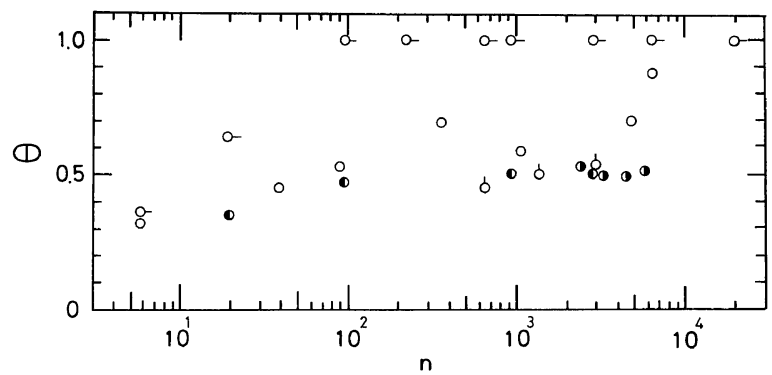

Figure 9. Plot of $\theta v s$. the degree of polymerization. Symbols are the same as in Figure 7.

fact that they give a universal plot for $\Gamma$ against the degree of polymerization is worth-noting. More data will be necessary to confirm the universality of this relation.

Figures 8 and 9 show the degree of polymerization dependence of the measured values of $p$ and $\theta$, respectively. Most of data points for $p$ under the theta condition can be superimposed on the master curve, but some $p$ values measured by Linden and Leemput are located above the master curve. The $p$ values for low degrees of polymerization in good solvents deviate from the master curve. The $\theta$ values are fairly scattered compared to those of $\Gamma$ and $p$; in particular, the data of Linden and Leemput are somewhat larger than our data.

The results of our research warrant the conclusion that universal curves can be obtaind for $\Gamma$ and $p$ against the degree of polymerization, in spite of the difference in polymer segment- and solventsilanol group interactions.

\section{REFERENCES}

1. L. H. Little, "Infrared Spectra of Adsorbed Species," Academic Press, New York, N.Y., 1966.

2. B. J. Fontana and J. R. Thomas, J. Phys. Chem., 65, 480 (1961).

3. C. Thies, P. Peyser, and R. Ullman, The Determination of Polymer Structure at a LiquidSolid Interface by Infared Analysis, in Proceedings of the 4th International Congress on Surface Active Substrates, Vol. II, Gordon and Breach, Science
Publishers, New York, N.Y., 1967, p 1041.

4. P. Peyser, D. J. Tutas, and R. R. Stromberg, J. Polym. Sci. A-1, 5, 651 (1967).

5. C. Thies, J. Phys. Chem., 70, 3783 (1966).

6. C. Thies, Macromolecules, 1, 335 (1968).

7. G. R. Joppien, Makromol. Chem., 175, 1931 (1974).

8. G. R. Joppien, Makromol. Chem., 176, 1129 (1975).

9. E. Diets, Makromol. Chem., 177, 2113 (1976).

10. J. C. Day and I. D. Robb, Polymer, 21, 408 (1980).

11. M. Korn and E. Killmann, J. Colloid Inrerface Sci., 76, 19 (1980).

12. K. I. Brebner, R. S. Chahal, and L. E. St-Pierre, Polymer, 21, 533 (1980).

13. K. I. Brebner, G. R. Brown, R. S. Chahal, and L. E. St-Pierre, Polymer, 22, 56 (1981).

14. C. V. Linden and R. V. Leemput, J. Colloid Interface Sci., 67, 48 (1978).

15. M. Kawaguchi, K. Hayakawa, and A. Takahashi, Polym. J., 12, 265 (1980).

16. M. Kawaguchi, T. Sano, and A. Takahashi, Polym. J., 13, 1019 (1981).

17. D. M. Griffiths, K. Marshall, and C. H. Rochester, J. Chem. Soc. Faraday Trans. 1, 70, 400 (1974).

18. R. J. Roe, J. Chem. Phys., 60, 4192 (1974).

19. A. Silberberg, J. Chem. Phys., 48, 2835 (1968).

20. J. M. H. M. Scheutjens and G. J. Fleer, J. Phys. Chem., 83, 1619 (1979).

21. J. B. Berkowitz, M. Yamin, and R. M. Fuoss, J. Polym. Sci., 28, 69 (1958).

22. H. P. Boehm and M. Z. Schneider, Anorg. Allgem. Chem., 301, 326 (1959).

23. R. J. Perkel and R. Ullman, J. Polym. Sci., 54, 127 (1961).

24. A. Takahashi, M. Kawaguchi, H. Hirota, and T. Kato, Macromolecules, 13, 884 (1980). 